\title{
Qualidade do preparado para bebida obtido a partir de polpa de juçara submetida ao tratamento térmico
}

\author{
Quality of the beverage preparation produced from juçara pulp \\ subjected to heat treatment
}

\section{Rodrigo Weigand Castro', Graciele da Silva Campelo Borges², Luciano Valdemiro Gonzaga', Deise Helena Baggio Ribeiro ${ }^{1 *}$}

${ }^{1}$ Universidade Federal de Santa Catarina (UFSC), Departamento de Ciência e Tecnologia de Alimentos, Florianópolis/SC - Brasil

Universidade Federal da Paraíba (UFPB), Centro de Tecnologia e Desenvolvimento Regional, João Pessoa/PB - Brasil

\section{*Corresponding Author}

Deise Helena Baggio Ribeiro, Universidade Federal de Santa Catarina (UFSC), Departamento de Ciência e Tecnologia de Alimentos, Rodovia Ademar Gonzaga, 1346, Itacorubi, CEP: 88034-001, Florianópolis/SC - Brasil, e-mail: deise.baggio@ufsc.br

Cite as: Quality of the beverage preparation produced from juçara pulp subjected to heat treatment. Braz. J. Food Technol. v. 19, e2015008, 2016.

Received: Mar. 11, 2015; Accepted: May 31, 2016

\section{Resumo}

As palmeiras do gênero Euterpe têm notável importância econômica e cultural, sendo que o seu valor se deve em parte às inúmeras formas de utilização, dentre as quais se destacam o consumo da bebida proveniente dos frutos e do palmito. Este estudo tem por objetivo avaliar a eficiência do tratamento térmico prévio nos frutos de juçara ( $E$. edulis Martius) utilizados para o processamento da polpa de juçara, visando à obtenção de um preparado para bebida, com qualidade nutricional e segura para o consumo. Os frutos foram selecionados, higienizados com hipoclorito de sódio (200 ppm/15 min) e submetidos a três tratamentos térmicos $\left(100{ }^{\circ} \mathrm{C} / 5 \mathrm{~s}, 80^{\circ} \mathrm{C} / 10 \mathrm{~s}\right.$ e $\left.80^{\circ} \mathrm{C} / 30 \mathrm{~s}\right)$. Depois do tratamento foram realizados o despolpamento e o congelamento, seguidos de armazenamento. Os resultados das análises microbiológicas demonstram que é necessário o tratamento térmico a $100^{\circ} \mathrm{C} / 5 \mathrm{~s}$ para atender aos parâmetros microbiológicos exigidos pela legislação para polpa de açaí, além de preservar o conteúdo de antocianinas totais e atividade antioxidante. Desta forma, o tratamento térmico dos frutos apresenta-se como um método viável e de baixo custo, contribuindo para a produção da bebida com qualidade e segurança.

Palavras-chave: Tratamento térmico; Micro-organismos; Antioxidantes; Segurança alimentar e nutricional; Euterpe edulis M.

\section{Summary}

Palms of the genus Euterpe have remarkable economic and cultural importance, their value residing, in part, from the numerous forms of use, amongst which one can highlight the consumption of the palm hearts and of a beverage made from their fruits. This study aims to evaluate the efficiency of a prior heat treatment of the juçara fruits ( $E$. edulis Martius) used in the processing of the pulp destined to produce a beverage preparation with high nutritional quality and safe for consumption. The fruits were sorted, sanitized with sodium hypochlorite $(200 \mathrm{ppm} / 15 \mathrm{~min})$ and subjected to three heat treatments $\left(100{ }^{\circ} \mathrm{C} / 5 \mathrm{~s}\right.$, $80^{\circ} \mathrm{C} / 10 \mathrm{~s}$ and $80^{\circ} \mathrm{C} / 30 \mathrm{~s}$ ). After the heat treatments, the fruits were pulped, followed by freezing of the pulp and storage at $-20^{\circ} \mathrm{C}$. The results of the microbiological analyses demonstrated that only the treatment at $100{ }^{\circ} \mathrm{C} / 5 \mathrm{~s}$ was adequate to conform with the microbiological parameters required by Brazilian legislation for açaí pulp, and also preserve the anthocyanin content and antioxidant activity. In conclusion, the heat treatment $\left(100{ }^{\circ} \mathrm{C} / 5 \mathrm{~s}\right)$ is a feasible, fast and low-cost method, which contributes to the production of a high quality and safe beverage.

Keywords: Heat treatment; Microorganisms; Antioxidant; Food safety; Euterpe edulis M. 


\section{Introdução}

Euterpe edulis Martius é uma palmeira conhecida popularmente por palmeira juçara. Sua distribuição estende-se desde o Estado da Bahia até o Estado do Rio Grande do Sul/BR, por quase toda a zona da mata pluvial atlântica, atingindo até 700 metros de altitude (REIS et al. 2000). Devido à exploração intensiva do palmito, sua ocorrência natural foi severamente reduzida a ponto de compor a lista de espécies brasileiras em extinção (BRASIL, 2008).

Na região Sul-Sudeste brasileira, a partir de 2003, o manejo de $E$. edulis M. passou a focar a produção de frutos popularmente chamados de "juçara", "jussara" ou "jiçara", notadamente como alternativa à exploração da espécie para extração do palmito (FARIAS, 2009).

Os frutos de juçara ( $E$. edulis M.) são matéria-prima para produção de polpa ou suco com alto teor lipídico, rico em ácidos graxos insaturados e compostos fenólicos, além de apresentar um elevado teor de antocianinas (BORGES et al., 2011a). Apesar de sua ampla distribuição no Brasil, os frutos de juçara são muito menos consumidos do que os de açaí (E. oleraceae) (BRITO et al., 2007).

Os frutos de juçara são despolpados, normalmente por maceração em água ou em uma despolpadeira mecânica, a fim de obter um produto roxo viscoso denominado polpa de juçara (BORGES et al., 2011a).

A polpa de juçara é altamente perecível, assim como a polpa de açaí, e esta condição pode ser agravada pela exposição a agentes microbianos conforme as práticas pós-colheita a que os frutos são submetidos, desde a extração dos frutos até o transporte e armazenamento. Além disso, no processo de obtenção da polpa, a manipulação favorece a proliferação de microrganismos e reações enzimáticas, principais responsáveis pela deterioração (ROGEZ, 2000).

Considerando que o processamento dos frutos de juçara ( $E$. edulis M.) ocorre principalmente em pequenas agroindústrias, a diversificação da produção é considerada uma alternativa de fonte de renda para a agricultura familiar. Atualmente, algumas agroindústrias empregam a pasteurização, na conservação da polpa de juçara, como uma forma de redução da carga microbiana, inativação enzimática e preservação das antocianinas (ROGEZ, 2000). Entretanto, segundo De Marchi (2001) apud Barros et al. (2015), durante o processamento e a estocagem da polpa, poderá ocorrer degradação com alteração de seu valor nutricional, do aroma e do sabor, dependendo da temperatura de estocagem, do oxigênio residual e da permeabilidade da embalagem ao oxigênio, entre outros fatores, que podem limitar sua vida útil. Outras agroindústrias familiares, devido ao alto custo para investimento de equipamentos, não utilizam nenhum tratamento térmico para conservação da polpa, o que compromete a segurança alimentar e nutricional do produto.

A adoção de procedimentos que visam à qualidade e à padronização da produção terá um impacto positivo no mercado, provendo, ao longo do tempo, um aumento da demanda, gerando desenvolvimento local e concomitante conservação da espécie. Também permitirá que se obtenha um alimento de alto valor nutricional e funcional, além de garantir a segurança microbiológica e o prolongamento da vida útil da polpa e dos produtos dela derivados.

Este trabalho teve por objetivo estabelecer as condições do tratamento térmico mínimo necessário para garantir a segurança do consumo do preparado para bebida de frutos de juçara (E. edulis M.), bem como avaliar seu efeito sobre alguns parâmetros físico-químicos, conteúdo de compostos fenólicos totais, antocianinas totais e atividade antioxidante.

\section{Material e métodos}

\subsection{Coleta dos frutos}

Os frutos de juçara ( $E$. edulis M.) foram coletados de cachos em estádio de maturação comercial de palmeiras localizadas no município de Santo Amaro da Imperatriz, no Estado de Santa Catarina/BR.

Depois da colheita, os cachos foram debulhados e, em seguida, foram selecionados os frutos quanto ao estádio de maturação comercial, totalizando, aproximadamente, 20 kg após o descarte dos frutos doentes ou injuriados e com sintomas de manchas cloróticas escuras ou avermelhadas.

Os frutos selecionados foram acondicionados em caixas plásticas arejadas e transportados para os Laboratórios de Microbiologia e Química de Alimentos do Departamento de Ciência e Tecnologia dos Alimentos - UFSC.

\subsection{Processamento da polpa e obtenção do preparado}

Os frutos foram quarteados em lotes de $2 \mathrm{~kg}$ e submetidos à sanitização com solução de hipoclorito de sódio a 200 ppm por 15 minutos. Na sequência, foram submetidos aos tratamentos térmicos, conforme Figura 1. Os tratamentos térmicos utilizados seguiram três combinações tempo $\mathrm{x}$ temperatura, resultando nas seguintes condições de branqueamento: $80^{\circ} \mathrm{C}$ por 10 segundos; $80^{\circ} \mathrm{C}$ por 30 segundos; e $100{ }^{\circ} \mathrm{C}$ por 5 segundos, realizados em banho-maria (Dubnhoff DI-921, Dist, Florianópolis, SC, Brasil). Em seguida, os frutos foram resfriados em recipiente contendo água e gelo, a fim de promover o choque térmico, evitando o amolecimento excessivo, além de perdas dos compostos de cor e de atividade antioxidantes. Para comparação com o tratamento 
Qualidade do preparado para bebida obtido a partir de polpa de juçara submetida ao tratamento térmico Castro, R. W. et al.

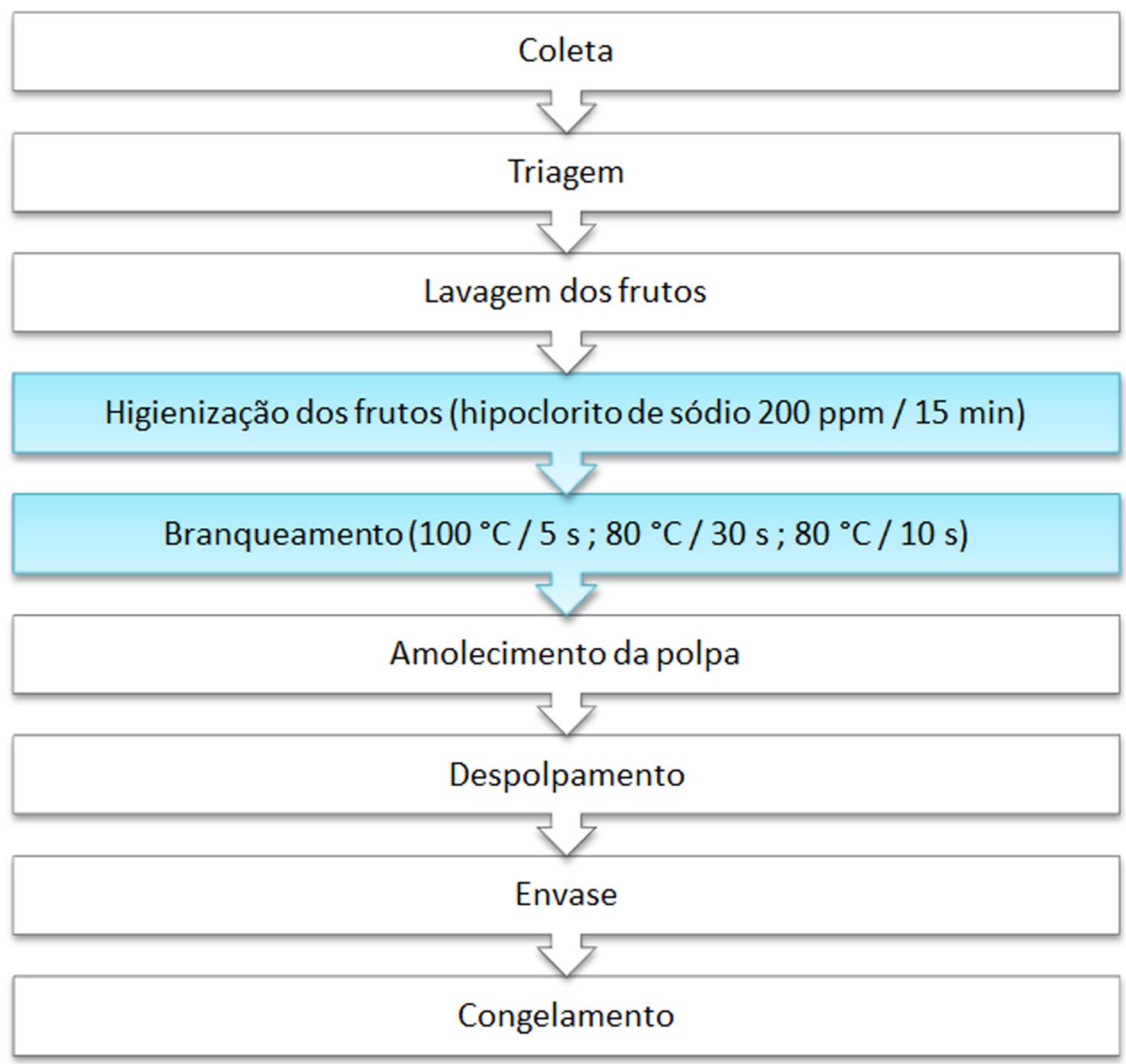

Figura 1. Fluxograma de processamento da polpa de juçara (Euterpe edulis M.).

térmico, utilizou-se uma amostra controle, a qual não foi submetida a tratamento térmico.

Depois do tratamento térmico, as amostras foram imersas em água mineral e mantidas a $40^{\circ} \mathrm{C}$ por 30 minutos, para amolecimento do pericarpo e do mesocarpo. A adição de água mineral baseou-se no fato de esta atender aos padrões de potabilidade e assim não ser um veículo de recontaminação. O despolpamento foi realizado em despolpadeira vertical, desenvolvida pelos próprios pesquisadores. O produto foi coletado e fracionado em sacos de polietileno e submetido ao congelamento em câmara fria a $-20^{\circ} \mathrm{C}$ (Santana, Araguaína, TO, Brasil), até o momento das análises.

\subsection{Análises microbiológicas}

As análises microbiológicas foram realizadas segundo as metodologias propostas por Silva et al. (2010) para bolores e leveduras, coliformes totais e termotolerantes,
E. coli e Salmonella sp. As amostras que apresentaram desempenho satisfatório nas análises microbiológicas foram submetidas às análises físico-químicas e à determinação dos compostos fenólicos totais, bem como de atividade antioxidante e conteúdo total de antocianinas monoméricas.

\subsection{Análises físico-químicas}

As análises físico-químicas foram realizadas de acordo com métodos da AOAC (HORWITZ, 2005), avaliando-se o teor de umidade (925.09); $\mathrm{pH}$; teor de proteína (920.87); resíduo mineral fixo (923.03); acidez total titulável (942.15).

\subsection{Determinação do conteúdo de compostos fenólicos totais e atividade antioxidante}

Para determinação do conteúdo de compostos fenólicos totais e atividade antioxidante, prepararam-se extratos conforme o método descrito por Borges et al. (2011a). 
Massas de $1 \mathrm{~g}$ do preparado foram transferidas para tubos de polietileno com capacidade de $50 \mathrm{~mL}$, adicionados $10 \mathrm{~mL}$ de metanol:água (50: 50) (v/v), homogeneizadas e submetidas ao processo de extração em ultrassom por 30 minutos (USC-1400 Unique, São Paulo, Brasil), centrifugação a 2000 x g por 15 minutos (Fanen 280R, São Paulo, Brasil). Em seguida, o sobrenadante foi transferido para balão volumétrico de $25 \mathrm{~mL}$ e o resíduo centrifugado foi submetido a um novo processo de extração com adição de $10 \mathrm{~mL}$ de acetona:água (70:30) (v/v) em ultrassom por 30 minutos. Novamente o extrato foi submetido ao processo de centrifugação a $2000 \mathrm{~g}$. Os extratos resultantes foram acumulados no balão volumétrico de $25 \mathrm{~mL}$ e o volume ajustado com água ultrapura.

O conteúdo de fenólicos totais dos extratos foi determinado pelo método espectrofotométrico com reativo de Folin-Ciocalteau (SINGLETON; ROSSI JUNIOR, 1965), com leituras realizadas a $765 \mathrm{~nm}$ em espectrofotômetro UV-Vis, modelo HP 8452A Hewlett-Packard (Cheadle Heath, Stockport Cheshire, UK). Os resultados foram expressos em miligramas de equivalentes a ácido gálico (EAG) $100 \mathrm{~g}^{-1}$ de polpa, calculados a partir da curva de calibração do ácido gálico (50-500 $\mu \mathrm{mol}$; y = 724,0x + $14,79, r^{2}=0,997 ; y=$ concentração da solução; $x$ = leitura da absorbância).

A avaliação da atividade antioxidante foi quantificada por meio da capacidade dos compostos presentes nos extratos em sequestrar/retardar o radical estável DPPH', de acordo com o método descrito por Brand Williams et al. (1995). A solução de trabalho de DPPH com absorbância de 0,980 $\pm 0,02$ em $515 \mathrm{~nm}$ foi preparada a partir de uma solução estoque DPPH 0,06 mM DPPH em metanol $80 \%$. Uma alíquota de $2,9 \mathrm{~mL}$ da solução de trabalho de DPPH foi adicionada a uma cubeta (caminho ótico de $1 \mathrm{~cm}$ ) e realizada a leitura da absorbância a $515 \mathrm{~nm}\left(\mathrm{t}_{\text {omin }}\right)$. Posteriormente adicionaram-se $100 \mu \mathrm{L}$ do extrato de juçara. O percentual de inibição do radical foi medido pela leitura de absorbância em espectrofotômetro Hewlett-Packard modelo HP 8452A (Cheadle Heath, Stockport, Cheshire, UK) a $515 \mathrm{~nm}$, o radical antes de adicionados os extratos $\left(A_{0}\right)$ e após a adição $\left(A_{f}\right)$ do extrato com tempo de reação fixado em 30 minutos. Uma curva padrão de Trolox (75-1050 $\mu \mathrm{M}$; $y=14,089 x+10,858, r 2=0.9953 ; y=$ concentração da solução; $x$ = leitura da absorbância) foi utilizada para quantificar a atividade antioxidante dos extratos e os resultados expressos em $\mu \mathrm{M}$ capacidade antioxidante equivalente a Trolox (TEAC) $100 \mathrm{~g}^{-1}$ de polpa.

\subsection{Quantificação de antocianinas monoméricas totais}

Para a quantificação de antocianinas monoméricas totais, realizou-se a extração por maceração a frio de $2,5 \mathrm{~g}$ do preparado de juçara com $50 \mathrm{~mL}$ de metanol 1,5 M HCl em frasco âmbar, à temperatura de $5 \pm 2{ }^{\circ} \mathrm{C}$ por $24 \mathrm{~h}$
(BORGES et al., 2011b). Em sequência, os extratos foram centrifugados a $4000 \mathrm{~g}$ por 10 minutos, aferidos a volume de $50 \mathrm{~mL}$. A quantificação foi realizada pelo método de diferença de pH descrito por Giusti e Wrolstad (2001), com leituras das absorbâncias a 510 nm e a 700 nm, usando um espectrofotômetro Hewlett-Packard, modelo HP 8452A (Cheadle Heath, Stockport, Cheshire, UK). O conteúdo de antocianinas monoméricas totais foi expresso em mg cianidina3-glicosídeo/100 g de polpa.

\subsection{Análise estatística}

Todas as amostras do preparado de juçara foram obtidas em duplicatas e as determinações analíticas foram realizadas em triplicatas. Os resultados foram expressos como a média \pm desvio padrão e submetidos à análise de variância (ANOVA), seguida do teste de t-Student para comparação de médias, ao nível de significância de 5\%.

\section{Resultados e discussão}

A legislação brasileira, na sua Instrução Normativa $n^{\circ} 1$ de 7/1/2000 do Ministério da Agricultura, Pecuária e Abastecimento (BRASIL, 2000), fixa o Padrão de Identidade e Qualidade (PIQ) para o açaí obtido de E. oleracea Mart. Apesar de a legislação não contemplar produtos similares que podem ser obtidos a partir de E. precatoria (ROGEZ, 2000) e de E. edulis (MAC FADDEN, 2005), neste trabalho, os valores estabelecidos nesta legislação foram considerados como referência.

As análises microbiológicas foram realizadas em duas amostras independentes de cada tratamento. A Tabela 1 apresenta os resultados obtidos e os valores padrões, considerando como referência a Instrução Normativa $n^{\circ} 1$ de 7/1/2000 do MAPA (BRASIL, 2000). Observou-se que a redução da carga microbiana foi diretamente proporcional à intensidade do tratamento térmico.

O tratamento de $80^{\circ} \mathrm{C}$ por 10 segundos não foi capaz de eliminar os coliformes termotolerantes, além de apresentar contagem de bolores e leveduras superior ao estabelecido na IN 01/2000. Apesar da notável redução da carga microbiana, o tratamento a $80^{\circ} \mathrm{C}$ por 30 segundos também não foi suficiente para reduzir a microbiota aos níveis aceitáveis. Quando comparados estes dados com os valores obtidos por Rogez (2000), analisando $E$. oleracea, nota-se que a redução de uma ordem logarítmica, utilizando $80^{\circ} \mathrm{C}$ por 10 segundos, só foi atingida no experimento com o aumento do tempo para 30 segundos de tratamento térmico.

No tratamento a $100{ }^{\circ} \mathrm{C}$ por 5 segundos, foi observada redução superior a 81\% para coliformes totais e superior a $99 \%$ para bolores e leveduras. Esse resultado indica a viabilidade do uso deste tratamento no controle microbiológico do extrato aquoso dos frutos e que ele proporciona um produto adequado para o consumo humano, conforme padrões legais vigentes. 
Tabela 1. Contagens microbianas do preparado de juçara ( $E$. edulis M.), submetido a diferentes tratamentos térmicos.

\begin{tabular}{|c|c|c|c|c|c|}
\hline Tratamento & Amostra & Salmonella spp & $\begin{array}{l}\text { Coliformes Totais } \\
\qquad\left(\text { NMP } \cdot g^{-1}\right)\end{array}$ & $\begin{array}{c}\text { Coliformes } \\
\text { termotolerantes } \\
\left(\text { NMP } \cdot \mathbf{g}^{-1}\right)\end{array}$ & $\begin{array}{l}\text { Bolores e } \\
\text { Leveduras } \\
\left(\text { UFC . } \text { g }^{-1}\right)\end{array}$ \\
\hline \multirow{2}{*}{ Controle } & 1 & Ausente & 16 & $<3$ & $2,3 \times 10^{3}$ \\
\hline & 2 & Ausente & 16 & $<3$ & $3,1 \times 10^{3}$ \\
\hline \multirow{2}{*}{$100^{\circ} \mathrm{C} / 5 \mathrm{~s}$} & 1 & Ausente & $<3$ & $<3$ & $5,0 \times 10^{1}$ \\
\hline & 2 & Ausente & $<3$ & $<3$ & $<1 \times 10^{1}$ \\
\hline \multirow{2}{*}{$80^{\circ} \mathrm{C} / 30 \mathrm{~s}$} & 1 & Ausente & 11 & $<3$ & $6,0 \times 10^{2}$ \\
\hline & 2 & Ausente & 3,6 & $<3$ & $5,5 \times 10^{2}$ \\
\hline \multirow{2}{*}{$80^{\circ} \mathrm{C} / 10 \mathrm{~s}$} & 1 & Ausente & 3 & 3 & $1,5 \times 10^{2}$ \\
\hline & 2 & Ausente & $<3$ & $<3$ & $5,7 \times 10^{3}$ \\
\hline IN 1/2000* & & Ausente & $<3$ & $<3$ & $2,0 \times 10^{3}$ \\
\hline
\end{tabular}

*Brasil (2000).

No que diz respeito à eficiência na redução da contaminação microbiológica, foi observado que o tratamento térmico aplicado aos frutos apresentou resultados similares ou superiores aos obtidos por outros autores quando o mesmo processo foi utilizado diretamente na polpa (SOUSA et al., 2006; ALEXANDRE et al., 2004; ROSENTHAL et al., 2006; ROGEZ, 2000).

Os resultados encontrados estão diretamente relacionados com a baixa carga microbiana inicial, indicada pelo tratamento controle. Isto é mais bem observado ao se comparar os dados de Sousa et al. (2006) e Rogez (2000), que encontraram não somente alta carga microbiana inicial, mas resultados positivos para Escherichia coli, Staphylococcus aureus e Salmonella sp. em açaís comercializados em feiras na cidade de Belém/Pará/BR.

O cuidado com a integridade e a seleção dos frutos também têm efeitos importantes no controle da contaminação. A integridade está relacionada à técnica de colheita e debulha, assim como às condições de transporte dos frutos. As injúrias podem acelerar a perda de água, estimular a respiração dos frutos, acelerar a perda dos compostos oxidáveis, além de permitir o acesso de micro-organismos ao interior do fruto (ROGEZ, 2000). Por sua vez, a seleção tem como um dos objetivos evitar a dispersão da microbiota estabelecida em frutos doentes para aqueles sadios ou com pequenas injúrias bem como para o produto final, durante o processamento.

Nesse sentido, os dados aqui apresentados demonstram que os procedimentos pós-colheita adotados ocasionaram baixa contaminação dos frutos. Também contribuíram para o resultado, o cuidado com a higiene pessoal e dos instrumentos durante o manuseio e acondicionamento dos frutos e, especialmente, a etapa preliminar de sanitização dos frutos. A sanitização foi realizada com hipoclorito de sódio, um agente de grande poder bactericida e fungicida, recomendado na desinfecção de frutas e hortaliças para consumo in natura, conforme normas técnicas do Codex Alimentarius publicadas pela OMS e legitimadas pela RDC $\mathrm{n}^{\circ} 218$, de 29 de julho de 2005, que aprova o Regulamento técnico de Procedimentos Higiênico-Sanitários para Manipulação de Alimentos e Bebidas Preparados com Vegetais (BRASIL, 2005).

Os resultados das análises do pH, acidez titulável, teor de proteína e cinzas estão apresentados na Tabela 2.

A concentração dos sólidos totais do produto elaborado foi abaixo de $8 \%$, por isso não pode ser enquadrado na categoria de açaí fino, conforme especificado na Instrução Normativa 01/2000 (BRASIL, 2000), no entanto os demais parâmetros foram comparados com o desta categorização.

Em relação ao pH, os valores médios obtidos são próximos daqueles observados por Rogez (2000) e por Sousa et al. (2006), de 5,2 e 5,4, respectivamente.

O conteúdo de proteína total determinado neste experimento está de acordo com o padrão legal vigente que preconiza o teor mínimo de $6 \%$ de matéria seca (BRASIL, 2000) e está semelhante aos valores descritos por Borges (2010), de 5,13\% a 8,21\% em matéria seca de frutos de E. edulis. Valores similares foram observados por Schauss et al. (2006) em açaí obtido a partir de E. oleracea, de 7,59\% da matéria seca.

O teor de minerais do preparado, obtido a partir de frutos branqueados, de 2,88\% a 3,68\%, mostrou-se similar aos valores descritos por Borges (2010), que variam entre 1,55\% e 3,39\%. Em caracterização centesimal de açaí de E. oleracea, Pereira et al. (2002) obtiveram teor de 4,19\% de cinzas em relação à matéria seca.

De acordo com os resultados descritos na Tabela 3, não ocorreram perdas significativas no teor de antocianinas totais para a polpa de juçara submetida ao tratamento térmico dos frutos, comparando com a polpa cujos frutos não sofreram tratamento térmico. A aplicação de um tratamento térmico rápido nos frutos objetivou a redução da carga microbiana e a desnaturação das enzimas, somadas ainda à remoção do oxigênio dos espaços intracelulares, principalmente na parte mais superficial do fruto, evitando 
Tabela 2. Composição físico-química do preparado de juçara (E. edulis M.).

\begin{tabular}{|c|c|c|c|c|c|}
\hline Tratamento & $\begin{array}{l}\text { Sólidos Totais } \\
\text { (média } \pm \text { DP) }\end{array}$ & $\begin{array}{c}\text { pH } \\
(\text { média } \pm \text { DP) }\end{array}$ & $\begin{array}{c}\text { Acidez } \\
\text { Titulável } \\
\text { (média } \pm \text { DP) }\end{array}$ & $\begin{array}{c}\text { Proteínas } \\
(\% \text { MS) } \\
\text { (média } \pm \text { DP) }\end{array}$ & $\begin{array}{c}\text { Cinzas } \\
(\% \text { MS) } \\
\text { (média } \pm \text { DP) }\end{array}$ \\
\hline Controle & $5,14^{a} \pm 0,41$ & $5,13^{a} \pm 0,05$ & $0,14^{b} \pm 0,05$ & $8,44^{a} \pm 0,12$ & $4,95^{a} \pm 0,28$ \\
\hline $100{ }^{\circ} \mathrm{C} / 5 \mathrm{~s}$ & $6,92^{\mathrm{a}} \pm 1,44$ & $5,02^{b} \pm 0,02$ & $0,17^{a} \pm 0,05$ & $7,95^{a} \pm 0,12$ & $3,24^{b} \pm 0,44$ \\
\hline
\end{tabular}

Médias ( \pm desvio padrão) seguidas da mesma letra não diferem pelo teste-t a 5\% de significância. Acidez Titulável (mg de ácido cítrico/100 g de matéria). MS (matéria seca).

Tabela 3. Conteúdo de antocianinas, fenólicos totais e atividade antioxidante do preparado de juçara (E. edulis M.).

\begin{tabular}{|c|c|c|c|}
\hline Tratamento & $\begin{array}{c}\text { Antocianinas } \\
\left.\text { (mg de cia-3-gli } 100 \mathrm{~g}^{-1}\right) \\
\text { (média } \pm \text { DP) }\end{array}$ & $\begin{array}{l}\text { Fenólicos totais } \\
\text { (mg EAG } 100 \mathrm{~g}^{-1} \text { ) } \\
\text { (média } \pm \mathrm{DP} \text { ) }\end{array}$ & $\begin{array}{c}\text { Atividade antioxidante } \\
\text { (média } \pm \text { DP) }\end{array}$ \\
\hline Controle & $92,58^{a} \pm 4,06$ & $428,58^{a} \pm 9,46$ & $4,32^{b} \pm 0,41$ \\
\hline $100^{\circ} \mathrm{C} / 5 \mathrm{~s}$ & $95,63^{a} \pm 3,30$ & $382,98^{b} \pm 19,57$ & $5,28^{a} \pm 0,41$ \\
\hline
\end{tabular}

Médias ( \pm desvio padrão) seguidas da mesma letra, na mesma coluna, não diferem pelo teste-t a $5 \%$ de significância. *Valores expressos em capacidade antioxidante equivalente a Trolox por g de preparado ( $\mu$ Mol Trolox g-1). EAG: equivalente a ácido gálico.

assim as reações de oxidação enzimática. Tais reações têm como consequência a oxidação das antocianinas presentes nos frutos. Tendo em vista que as antocianinas estão localizadas nos vacúolos das células hipodérmicas, próximas à superfície, como alegam Pompeu et al. (2009).

Rogez (2000) observou comportamento semelhante estudando o branqueamento de frutos de E. oleracea. Segundo o autor, o branqueamento severo promove a retenção de pigmentos, principalmente em função da duração do tratamento. Schultz (2008), em processamento caseiro dos frutos de $E$. edulis, encontrou no produto valores de antocianinas que variaram em amostras in natura, de

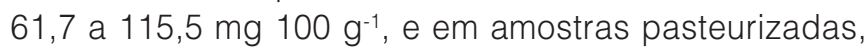
de 78,6 a 231,6 mg $100 \mathrm{~g}^{-1}$, equivalentes a cianidina 3-glicosídeo. O mesmo autor observou valores entre 13,2 e 34,9 mg $100 \mathrm{~g}^{-1}$ e entre 52,7 e 68,3 mg $100 \mathrm{~g}^{-1}$ em amostras de açaí produzido em agroindústria. Em açaí de E. oleracea, foram observados os valores entre 18,4 e 104,0 mg $100 \mathrm{~g}^{-1}$ (ROGEZ, 2000; HASSIMOTTO, 2005; KUSKOSKI et al., 2006; ALBARICI et al., 2007; SCHULTZ, 2008).

Mesmo com uma redução de $11 \%$ dos compostos fenólicos totais nos dados apresentados neste trabalho, pode-se considerar que o tratamento empregado teve um baixo impacto na atividade antioxidante. De acordo com Borges et al. (2011a), a atividade antioxidante dos frutos de juçara tem correlação com os compostos fenólicos totais. Em caracterização dos compostos fenólicos dos frutos de E. edulis, Borges et al. (2011a) observou que a quercetina representa $29 \%$ do conteúdo total, ficando apenas atrás do ácido gálico, que corresponde a 31\%. Dessa forma, o resultado observado pode indicar que o tratamento térmico não foi agressivo o suficiente para desencadear a degradação das antocianinas, somente afetando os compostos fenólicos.
Em relação aos compostos fenólicos, os valores observados, de 405,8 \pm 28,5 mg $100 \mathrm{~g}^{-1}$ EAG, estão em acordo com os resultados registrados na literatura para E. edulis M., mas acima dos valores para E. olerecea. Schultz (2008) encontrou para $E$. edulis valores de 480,4 a 653,3 mg $100 \mathrm{~g}^{-1}$ EAG e de 372,6 a $541,7 \mathrm{mg}$ $100 \mathrm{~g}^{-1}$ EAG em processo de extração caseiro com e sem pasteurização, respectivamente. A atividade antioxidante do preparado obtido após tratamento térmico dos frutos foi $22 \%$ maior do que no controle. Contudo, a atividade antioxidante de 4,8 \pm 0,6 $\mu \mathrm{Mol}$ de Trolox $\mathrm{g}^{-1}$ é inferior àquela observada por outros autores (SCHULTZ, 2008; BORGES et al., 2011a).

Por outro lado, Melo et al. (2008) consideram que a atividade antioxidante das frutas depende do teor de compostos fenólicos totais. Porém podem ser observadas variações quantitativas e qualitativas nesses compostos em função de fatores intrínsecos e extrínsecos, incluindo o grau de maturação dos frutos até a metodologia de quantificação. Esta conclusão também foi mencionada por Borges et al. (2011a) que concluem que o solvente utilizado, o tempo de extração e a razão sólido/solvente influenciam a performance da extração e posterior determinação em espectrofotômetro.

\section{Conclusões}

Conforme avaliação microbiológica, o tratamento térmico dos frutos de Euterpe edulis M. a $100{ }^{\circ} \mathrm{C}$ por 5 segundos demonstrou ser eficaz na obtenção de um produto microbiologicamente seguro, com poucas alterações nas características físico-químicas avaliadas e pequena degradação de compostos fenólicos totais. Além disso, não provocou alterações no teor de antocianinas e apresentou aumento da atividade antioxidante. O processo pode facilmente ser adaptado para pequenas agroindústrias. 
Qualidade do preparado para bebida obtido a partir de polpa de juçara submetida ao tratamento térmico Castro, R. W. et al.

\section{Referências}

ALBARICI T. R.; VAlETA A. C. ; PESSOA J. D. C. Efeito da temperatura nas antocianinas de açaí. São Carlos: EMBRAPA, 2007. (Comunicado Técnico; 86).

ALEXANDRE, D.; CUNHA, R. L.; HUBINGER, M. D. Conservação do açaí pela tecnologia de obstáculos. Ciência e Tecnologia de Alimentos, Campinas, v. 24, n. 1, p. 114-119, 2004. http:// dx.doi.org/10.1590/S0101-20612004000100021.

BARROS, E. C. M.; COSTA, G. N. S.; RIBEIRO, L. O.; MENDES, M. F.; PEREIRA, C. S. S. Effects of pasteurization on the physicochemical microbiological and anthocyanins from juçaí pulp (Euterpe edulis Martius). Revista Teccen, Vassouras, v. 8, n. 1, p. 1-32, 2015.

BORGES, G. S. C. Caracterização química e avaliação da atividade antioxidante in vitro de frutos de jussara (Euterpe edulis). 2010. 119 f. Dissertação (Mestrado em Ciências dos Alimentos)-Universidade Federal de Santa Catarina, Florianópolis, 2010.

BORGES, G. S. C.; VIEIRA, F. G. K.; COPETTI, C.; GONZAGA, L. V.; ZAMBIAZI, R. C.; MANCINI FILHO, J.; FETT, R. Chemical characterization bioactive compounds and antioxidant capacity of jussara (Euterpe edulis) fruit from the Atlantic Forest in southern Brazil. Food Research International, Oxford, v. 44, n. 7, p. 21282133, 2011a. http://dx.doi.org/10.1016/j.foodres.2010.12.006.

BORGES, G. S. P.; VIEIRA, F. G. K.; COPETTI, C.; GONZAGA, L. V.; FETT, R. Optimization of the extraction of flavanols and anthocyanins from the fruit pulp of Euterpe edulis using the response surface methodology. Food Research International, Oxford, v. 44, n. 3, p. 708-715, 2011b. http://dx.doi.org/10.1016/j. foodres.2010.12.025.

BRAND-WILLIAMS, W.; CUVELIER, M. E.; BERSET, C. Use of a free radical method to evaluate antioxidant activity. LWT - Food Science and Technology, London, v. 28, p. 25-30, 1995.

BRASIL. Ministério da Agricultura da Pecuária e do Abastecimento. Instrução Normativa $n^{\circ}$ 01, de 7 de janeiro de 2000. Regulamento técnico para fixação dos padrões de identidade e de qualidade para polpas de frutas. Diário Oficial [da] República Federativa do Brasil, Brasília, DF, 2000.

BRASIL. Agência Nacional de Vigilância Sanitária. Resolução $\mathrm{RDC} \mathrm{n}^{\circ} 218$, de 29 de julho de 2005. Aprova regulamento técnico de procedimentos higiênico-sanitários para manipulação de alimentos e bebidas preparados com vegetais. Diário Oficial [da] República Federativa do Brasil, Brasília, DF, 2005.

BRASIL. Ministério do Meio Ambiente. Instrução Normativa $n^{\circ} 6$ de 23, de setembro de 2008. Lista oficial das espécies da flora brasileira ameaçadas de extinção. Diário Oficial [da] República Federativa do Brasil, Brasília, DF, 2008.

BRITO, E. S.; ARAÚJO, M. C. P.; ALVES, R. E.; CARKEET, C. C.; CLEVIDENCE, B.; NOVOTNY, J. Anthocyanins present in selected tropical fruits: Acerola, jambolão, Jussara e guarabiju. Journal of Agricultural and Food Chemistry, v. 55, n. 23, p. 9389-9394, 2007. http://dx.doi.org/10.1021/jf0715020. PMid: 17929888.

FARIAS, M. Reinventando a relação humano - Euterpe edulis: do palmito ao açaí. 2009. 85 f. Dissertação (Mestrado em Agroecossistemas)-Universidade Federal de Santa Catarina, Florianópolis, 2009

GIUSTI, M. M.; WROLSTAD, R. E. Antocyanins: characterization and measurement with uv-visible spectroscopy. In: WROSTAD, R. E. Current protocols in food analytical chemistry. New York: John Wiley \& Sons, 2001. p. 1-13.

HASSIMOTTO, N. M. A. Atividade antioxidante de alimentos vegetais: estrutura e estudo de biodisponibilidade de antocianinas de amora silvestre (Morus sp.). 2005. 159 f. Tese (Doutorado em bromatologia)-Faculdade de Ciências Farmacêuticas, Universidade de São Paulo, São Paulo, 2005.

HORWITZ, W. (Ed.). Official methods of analysis of the Association of Official Analytical Chemists. 18th ed. Gaithersburg: AOAC, 2005. $1260 \mathrm{p}$.

KUSKOSKI, E. M.; ASUERO, A. G.; MORALES, M. T.; FETT, R. Frutos tropicais silvestres e polpas de frutas congeladas: atividade antioxidante polifenóis e antocianinas. Ciência Rural, Santa Maria, v. 36, n. 4, p. 1283-1287, 2006. http://dx.doi.org/10.1590/ S0103-84782006000400037.

MAC FADDEN, J. A produção de açaí a partir do processamento dos frutos do palmiteiro (Euterpe edulis Martius) na Mata Atlântica. 2005. 100 f. Dissertação (Mestrado em Agroecossistemas)Universidade Federal de Santa Catarina, Florianópolis, 2005.

MELO, E. A.; MACIEL, M. I. S.; LIMA, V. L. A. G.; NASCIMENTO, R. J. Capacidade antioxidante de frutas. Revista Brasileira Ciências Farmacêuticas, São Paulo, v. 44, n. 2, p. 193-201, 2008. http://dx.doi.org/10.1590/S1516-93322008000200005.

PEREIRA, E. A.; QUEIROZ, A. J. M.; FIGUEIRÊDO, R. M. F. Massa específica de polpa de açaí em função do teor de sólidos totais e da temperatura. Revista Brasileira de Engenharia Agrícola e Ambiental, Campina Grande, v. 6, n. 3, p. 526-530, 2002. http:// dx.doi.org/10.1590/S1415-43662002000300025.

POMPEU, D. R.; SILVA, E. M.; ROGEZ, H. Optimization of the solvent extraction of phenolic antioxidants from fruits of Euterpe oleracea using response surface methodology. Bioresource Technology, New York, v. 100, n. 23, p. 6076-6082, 2009. http:// dx.doi.org/10.1016/j.biortech.2009.03.083. PMid:19581082.

REIS, M. S.; FANTINI, A. C.; NODARI, R. O.; GUERRA, M. P.; REIS, A. Sustained yield management of Euterpe edulis Martius (Palmae): a tropical palm tree from the Atlantic Tropical Forest. Journal of Sustainable Forestry, New Haven, v. 11, n. 3, p. 1-17, 2000. http://dx.doi.org/10.1300/J091v11n03_01.

ROGEZ, H. Açaí: preparo composição e melhoramento da conservação. Belém: EDUFPA, 2000. 313 p. 
Qualidade do preparado para bebida obtido a partir de polpa de juçara submetida ao tratamento térmico Castro, R. W. et al.

ROSENTHAL A.; SIQUEIRA R. S.; MENEZES E. M. S.; SRUR A. S.; CAMARGO L. M. A. Q.; DELIZA R. Processamento de polpa de açai por alta pressão hidrostática. Rio de Janeiro: Embrapa Agroindústria de Alimentos, 2006. (Comunicado técnico; 103).

SCHAUSS, A. G.; WU, X.; PRIOR, R. L.; OU, B.; PATEL, D.; HUANG, D.; KABABICK, J. P. Antioxidant capacity and others bioactivities of the freeze-dried Amazonian palm berry Euterpe oleracea Mart. (açaí). Journal of Agricultural and Food Chemistry, Washington, v. 54, n. 22, p. 8598-8603, 2006. http:// dx.doi.org/10.1021/jf060976g. PMid:17061839.

SCHULTZ, J. Compostos fenólicos antocianinas e atividade antioxidante de açaí de Euterpe edulis Martius e Euterpe oleracea Martius e influência de diferentes métodos de pasteurização sobre o açaí de Euterpe edulis. 2008. 53 f.
Trabalho de Conclusão de Curso (Graduação em Agronomia)Universidade Federal de Santa Catarina, Florianópolis, 2008.

SILVA N.; JUNQUEIRA V.; SILVEIRA N. F. A.; TANIWAKI M. H.; SANTOS R. F. S.; GOMES, R. A. R. Manual de métodos de análise microbiológica de alimentos. 4. ed. São Paulo: Varela, 2010. SINGLETON, V. L.; ROSSI JUNIOR, J. A. Colorimetry of total phenolics with phosphomolybdic phosphotungstic acid reagents. American Journal of Enology and Viticulture, Davis, v. 16, p. 144-158, 1965.

SOUSA, M. A. C.; YUYAMA, L. K. O.; AGUIAR, J. P. L.; PANTOJA, L. Suco de açaí (Euterpe Oleracea Mart.): avaliação microbiológica tratamento térmico e vida de prateleira. Acta Amazonica, Manaus, v. 36, n. 4, p. 483-496, 2006. http://dx.doi.org/10.1590/ S0044-59672006000400010. 